\title{
An Experimental Evaluation of Robustness of Networks
}

\author{
Hyoungshick Kim and Ross Anderson
}

\begin{abstract}
Models of conflict in networks provide insights into applications from epidemiology to guerilla warfare. Barabási, Albert and Jeong modelled selective attacks on networks in which an attacker targets high-order nodes to destroy connectivity; Nagaraja and Anderson extended this to iterated attacks where the attacker and defender take turns to remove and rebuild nodes and edges according to given strategies. We extend the iterative model by introducing the cost required to perform network operations. This gives much finer granularity than previous models, whether we are interested in network resilience against random failures or intentional attacks. We empirically study how to design more effective attacks and/or defenses through intensive simulation on several well-known network topologies including the three real-world networks. In particular, an effective defence against many attacks is to add new links connecting low-centrality nodes to maintain the overall balance of network centrality.
\end{abstract}

Index Terms-Network Robustness; Network Resilience; Iterative Attacks and Defenses

\section{INTRODUCTION}

Many important phenomena depend on networks, from social interactions between people to explicit networks such as the Internet and supply chains. Recent advances in the theory of networks have provided us with mathematical and computational tools to understand them better [1], [2]. Often the topology of a network has distinctive features, such as vertex order distribution, clustering and characteristic path lengths, which can be explained in terms of its evolution and which in turn explain some aspects of its behaviour. For example, networks that grow by preferential attachment may acquire a power-law distribution of vertex order that in turn makes them robust against random node failures - yet this distribution also makes them vulnerable to attacks targeted on high-degree nodes. Insights like this can inform activities from epidemiology to policing. Doctors may first vaccinate those individuals who are likely to come into contact with most others, while police forces tackle criminal gangs by placing the most highly connected criminals under arrest or surveillance. They also apply to technological networks such as the Internet, the electrical power grid and transportation networks; these are also robust to random failures but vulnerable to targeted attacks [3].

Network failure models are not limited to the one-shot case. When an attack occurs, a defender tries to minimize the damage by deploying new resources, while the attacker

Hyoungshick Kim is with University of British Columbia, Vancouver, Canada (E-mail: hyoung@ece.ubc.ca).

Ross Anderson is with the Computer Laboratory, University of Cambridge, Cambridge, United Kingdom. (E-mail: ross.anderson@cl.cam.ac.uk. may then follow through by causing further damage. In other words, we need to consider dynamic interaction between an attacker and a defender over multiple periods. Nagaraja and Anderson developed a framework to explore iterated "attack" and "defense" operations: an attacker removes $k_{a}$ nodes from a network at each attack round, and a defender adds $k_{d}$ nodes to the network at each defense round. The attacker's aim is to decrease network connectivity or efficiency, which can be measured as the size of the largest connected component or the average shortest path length in the network, while the defender's aim is the opposite [4]. This models how a network will likely evolve under continuous attack, based on evolutionary game theory [5]. It enables us to investigate what sort of attack and defence strategies might prevail in counterrevolutionary warfare: a conflict in which peacekeepers identify and arrest rebel ring leaders, while the rebels constantly recruit and reorganise themselves. However, Nagaraja and Anderson's work has two limitations: (1) it does not model the costs of creating new nodes and edges realistically, as the defender is allowed to create a fixed number of new nodes at each round plus an arbitrary number of edges. In practice the cost of establishing edges is not zero, so it would be preferable to enable the defender to allocate his budget to nodes and edges with some fixed marginal cost of substitution (2) their experimental results were limited to a single scenario, namely a Barabási-Albert scale-free network consisting of 400 nodes with most of the experiments involving $k_{a}=k_{d}=10$ and a few dozen rounds.

We extend their work into a more generalized model: While Nagaraja and Anderson simply assumed a newly recruited node could form the right number of new connections to pursue any given defense strategy, we vary the budgets to limit newly added nodes and their connections. We note that to make a network highly robust against node removal attacks, a simple strategy is just to increase its network density. In this paper, we seek to answer a simple question: "How much does a connection cost?" We want a quantitative understanding of the correlation between network density and resilience to random failures or attacks as well as insight into the evolution of networks by iterative growth and shrinkage processes.

We therefore empirically analyse the effects of attack/defense strategies with more realistic budgets on wellknown network topologies: two Erdős-Rényi random graphs, two Barabási-Albert scale free networks [6], two Chord networks [7], two Hypergrid graphs [8], a Transit-Stub graphs [9], a Watts-Strogatz small world network [10], a Content-Addressable network [11], a PRU network [12], and the three real-world networks [13], [14], [15]. 
Our experimental results show that the strategy of connecting low centrality nodes produces the best overall performance for maintaining network connectivity for a given budget. Also, even simple defense strategies (e.g. adding nodes and their connections randomly) can be effective enough to fight against sophisticated attacks (e.g. removing nodes with high centrality in high priority) if we can increase the number of connections per node past a certain threshold.

\section{RELATED WORK}

Albert, Jeong and Barabási [3] showed that attacks targeted on high-degree nodes in scale-free networks are much more effective than random attacks; the size of the largest connected component is rapidly reduced. This is because scale-free networks get much of their connectivity from few nodes of high degree. It is hard to remove enough of those hub nodes in a random attack, but if they are targeted deliberately, then connectivity decreases dramatically. Holme and others [16] got similar experimental results by doing such attacks on edges, and also suggested using centrality as an alternative to degree for targeting. Zhao and others [17] studied the circumstances under which a scale-free network suffers cascading breakdown caused by the successive failures of hub nodes.

Nagaraja and Anderson [4] extended this by introducing a framework from evolutionary game theory to explore the effectiveness of iterated attack and defense operations. They showed that a defender can make a network resilient to attacks by replacing highly connected nodes with cliques - small groups of vertices that are fully connected to each other and which share the outgoing edges that previously went to a single highly-connected node. This strategy, however, requires the modification of the existing connections in a network and may have high implementation costs if adding an extra edge is expensive. Clique-based defense strategies are likely to be nontrivial in some environments such as wired networks.

Recently, Domingo-Ferrer has been extending Nagaraja and Anderson's model to weighted and directed networks [18]. He also found that the costs of attacks/defenses were not clearly defined in [4] and discussed the economic aspects of the attack and defense strategies by considering the cost of node destruction/replenishment. However, it is hard to evaluate the usefulness of the estimated cost functions; as already noted, the cost of rewiring edges is usually not zero. We therefore set out to refine the iterated attack / defense model to take account of the cost of edges added or changed as well as the number of nodes added during the defense phase. We also extended the modelling to a much larger range of graph topologies.

\section{MODEL}

Our framework can formally be represented as a game on a graph $G$ by iterating "attack" and "defense" operations for a certain number of rounds. Here an attacker's objective is to maximize disruption to the network while a defender tries to minimize it.

Each round consists of an attack phase followed by a defense phase. In an attack phase, an attacker picks the existing $k_{a}$ nodes from the graph $G$ according to her attack strategy and then removes the selected nodes and their associated edges. In a defense phase, a defender creates $k_{d}$ new nodes and then adds them by sequentially connecting a new node $v$ with $m$ edges to $m$ different nodes already present in $G$ according to his strategy. Unlike Nagaraja and Anderson's model, we do not allow the defender to rewire the existing edges in the graph $G$ - this may be expensive compared to establishing new edges in some real environments such as wired networks since an edge rewiring operation can actually be treated as a combination of destroying existing edges and establishing new edges. We assume that the defender has no knowledge of which nodes and their connections are disappeared (otherwise the best strategy may be to restore the last status of the network when $k_{a}=k_{d}$ ).

In order to measure the effectiveness of attacks and defenses, we use the size of the largest connected component after a certain number of rounds as the metric, as other authors in this field have done.

\section{A. Attack strategies}

An attack strategy is a strategy (an algorithm) to select $k_{a}$ nodes to be removed from a graph $G=(V, E)$ in an attack phase. We here assume $k_{a}$ is a constant. We consider the following three strategies:

1) Random removal: Pick a node randomly from $G$ and remove it and its associated edges. Repeat this process $k_{a}$ times.

- This strategy is very simple and efficient: An attacker does not need any knowledge of the network topology. The total running time is $O(d(G))$ if we ignore the cost of random selection where $d(G)$ is the average node degree in the graph $G$.

2) High-degree removal: Pick the highest-degree node from $G$ and remove it and its associated edges. Repeat this process $k_{a}$ times.

- This strategy requires global knowledge of the node degree. The total running time is $O(|V| \log |V|)$ since the nodes are sorted in decreasing order with respect to their degree.

3) High-centrality removal: Pick the highest-betweenness centrality node from $G$ and remove it and its associated edges. Repeat this process $k_{a}$ times. Here, we only consider betweenness centrality since this is known to be more related to network connectivity than closeness or eigenvector centrality. Betweenness centrality $b(u)$ is calculated for a node $u$ as the proportion of shortest paths between all node pairs in the network that pass through $u$ :

$$
b(u)=\frac{1}{(|V|-1) \cdot(|V|-2)} \sum_{s \neq u, t \neq u \in V} \frac{\sigma_{s, t}(u)}{\sigma_{s, t}}
$$

where $\sigma_{s, t}$ is the total number of shortest paths from source node $s$ to destination node $t$, and $\sigma_{s, t}(u)$ is the number of shortest paths from source node $s$ to destination node $t$ which actually pass through node $u$.

- This strategy requires knowledge of the network topology and has total running time $O(|V| \cdot|E|+$ 
$|V| \log |V|$ ) (the nodes are sorted in decreasing order with respect to their betweenness centrality which can be computed in $O(|V| \cdot|E|)$ time [19].) We use $\mathbf{A}^{\text {random }}, \mathbf{A}^{\text {degree }}$, and $\mathbf{A}^{\text {central }}$ to denote random removal, high-degree removal, and high-centrality removal attack strategies, respectively. We note that all of these attack strategies remove the same number of $k_{a}$ nodes at every round if the remaining nodes of the graph $G$ is greater than or equal to $k_{a}$.

\section{B. Defense strategies}

A defense strategy is a strategy (algorithm) to connect $k_{d}$ newly recruited nodes to the existing nodes in a graph $G=$ $(V, E)$ in a defense phase. We here assume $k_{d}$ is a constant. A new node $v$ is connected with $m$ new edges to $m$ different nodes. We consider the following three strategies:

1) Random replenishment: Create a new node and add it to $G$ such that the node is connected with $m$ new edges to $m$ randomly selected different nodes. Repeat this process $k_{d}$ times.

- This strategy requires no knowledge of the network topology. The total running time is $O(m)$ if we ignore the cost of random selection.

2) Preferential replenishment: Create a new node and add it to $G$ such that the node is connected with $m$ new edges to $m$ different nodes with probability proportional to their degree (i.e. the node is connected to an existing node $u$ with the probability $p(u)=d(u) / \sum_{v \in V} d(v)$ where $d(u)$ is the node $u$ 's degree in $G$ ). Repeat this process $k_{d}$ times.

- This strategy requires knowledge of the node degree. The total running time is $O(|V|)$ if we ignore the cost of random selection.

3) Balanced replenishment: Create a new node and add it to $G$ such that the node is connected with $m$ new edges to $m$ different nodes with probability inversely proportional to their betweenness centrality (i.e. the node is connected to an existing node $u$ with the probability $p(u)=(b(u)+\epsilon)^{-1} / \sum_{v \in V}(b(v)+\epsilon)^{-1}$ where $b(u)$ is the node $u$ 's betweenness centrality in $G$ and $\epsilon$ is a very small constant to prevent division by zero). Repeat this process $k_{d}$ times.

- This strategy requires knowledge of the network topology. The total running time is $O(|V| \cdot|E|)$ if we ignore the cost of random selection.

We use $\mathbf{D}^{\text {random }}, \mathbf{D}^{\text {prefer }}$, and $\mathbf{D}^{\text {balance }}$ to denote random replenishment, preferential replenishment, and balanced replenishment defense strategies, respectively. We note that all of these defense strategies add the same numbers of $k_{d}$ nodes and $m \cdot k_{d}$ edges at every round.

\section{THE NECESSARY NETWORK DENSITY FOR ROBUST NETWORKS}

A fundamental question is whether we can make a network resilient against node failures or attacks by just increasing the number of edges.If a graph has too few edges, it is necessarily disconnected. As a network becomes better connected so its robustness will in general increase. And when providing network robustness we want to know the optimal edge budgets for newly recruited nodes.

To get an insight into this problem, we generated ErdôsRényi random graphs by varying the parameters (from 300 to 1,500 for the number of nodes $n$ and from 0 to 5 for the average node degree $d(G)$ ) and found the largest connected component in each graph. We repeated this 100 times for each tuple $(n, d(G))$ and computed the average size of the largest connected components over the sample. Figure 1 demonstrates the fraction of nodes remaining in the largest connected component with $d(G)$.

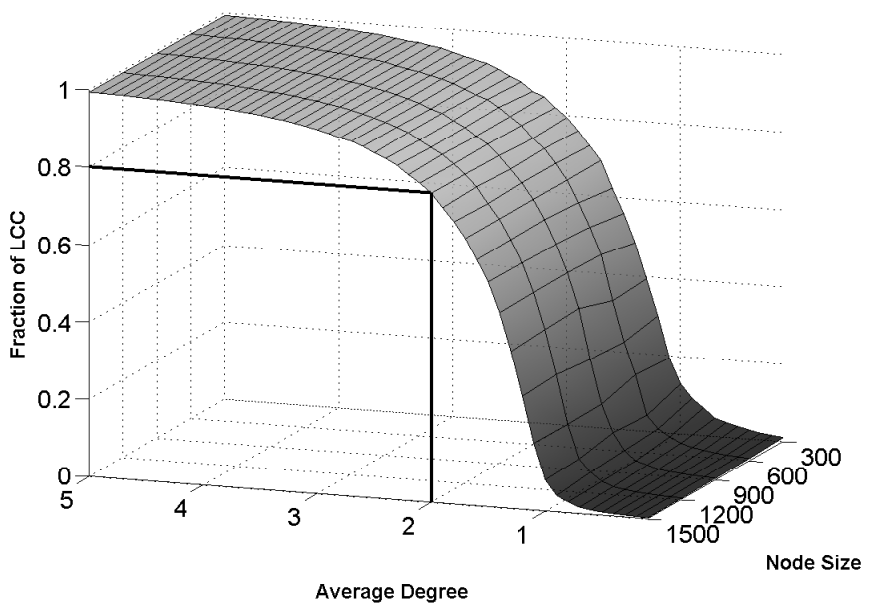

Fig. 1. The average size of the largest connected components over ErdôsRényi random graphs. With each pair of node size (from 300 to 1,500) and average degree (from 0 to 5) we generate 100 random graphs and compute the average size of the largest connected components over these graphs. All decay rates of the largest connected component in random graphs increases dramatically at $d(G)=2$.

The decay rates of the largest connected components in all Erdős-Rényi random graphs show almost the same pattern regardless of $n$. The curve has a gentle slope until $d(G)=2$ then plunges towards 0 when $d(G)<2$. As a selective attack is at least as effective as random edge removal, we can always expect a significant number of nodes to be disconnected from the network if $d(G)<2$. And when $d(G)=2$, the largest connected component in $G$ has a tendency to form a treelike graph structure which can be easily decomposable or be already reduced to a small component (see Figure 2).

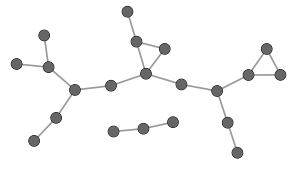

(a) Tree-like structure

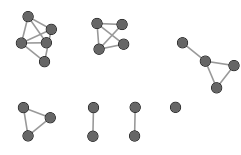

(b) Small components
Fig. 2. Example graphs with $n=21$ and $d(G)=2$. The largest connected component in the graph $G$ has (a) a tree-like graph structure or is already reduced to (b) a small component when $d(G)=2$.

In Section $\mathrm{V}$ we will explore the relationship between the size of the largest connected component and the average 
degree through intensive simulation results on various network topologies.

\section{Simulation Results}

Because of highly nonlinear characteristics of network structures, it is very difficult to establish mathematical models with a closed form solution. So, we use the simulation model as an alternative to the theoretical model. We experimentally tested the attack and defense strategies discussed in Section III-A and III-B on three real-world and twelve synthetic networks for evaluating the performance of the strategies as follows:

1) Random graphs $\left(G_{R}^{0.005}, G_{R}^{0.01}\right.$ - we denote as $G_{R}^{x}$ the random graph with the linking probability $x$ ): Random graphs are fundamental and useful for modelling problems in many applications.

2) Barabási-Albert scale free networks [6] $\left(G_{B A}^{2}, G_{B A}^{4}-\right.$ we denote as $G_{B A}^{x}$ the Barabási-Albert network where each new node is connected to $x$ existing nodes): Scalefree networks are abundant in nature and society, describing such diverse systems as the world wide web, the web of human sexual contacts, or the chemical network of a cell. Albert, Jeong and Barabási [3] showed that scale-free networks are resistant to random failures but vulnerable to targeted attacks since a few hubs dominate their topology.

3) Chord networks [7] $\left(G_{C}^{2}, G_{C}^{4}\right.$ - we denote as $G_{C}^{x}$ the Chord network where $x$ is the minimum degree of each node): Chord network is a typical structured peer-to-peer overlay network. Chord network is simple and useful to build a fault-tolerant and decentralized peer-to-peer structure.

4) Hypergrid graphs [8] $\left(G_{H}^{4}, G_{H}^{8}\right.$ - we denote as $G_{H}^{x}$ the Hypergrid network where $x$ is the maximum degree of each node): Hypergrid graph is built for peer-to-peer systems by enforcing low graph diameter and fixed node degree.

5) Transit-Stub graph [9] $\left(G_{T S}\right)$ : The Transit-Stub model is a hierarchical graph generation model that produces graphs having a structure similar to the Internet.

6) Watts-Strogatz small world network [10] $\left(G_{W S}^{4,0.1}-\right.$ we denote as $G_{W S}^{x, y}$ the Watts-Strogatz small world network with the initial $x$ neighbours and the linkino probability $y$ ): The Watts-Strogatz model is a rand graph generation model that produces graphs with sm world properties, including short average path len and high clustering.

7) Content-Addressable network [11] $\left(G_{C A}\right)$ : ContentAddressable network is designed for a distributed and scalable peer-to-peer systems.

8) PRU network [12] $\left(G_{P R U}^{50,12,2}\right.$ - we denote as $G_{P R U}^{x, y, z}$ the PRU network with the initial $c$ nodes in cache, the minimum degree $y$ of nodes and the maximum degree $z$ of nodes): PRU networks are suggested by Pandurangan et al. to produce graphs having a structure similar to the unstructured P2P networks.

9) Email network [13] $\left(G_{M A I L}\right)$ : The Email network is obtained by collecting mutual email communication interactions through email logs from a company.

10) Blog network [14] $\left(G_{B L O G}\right)$ : The Blog network is obtained by analysing the network structure of political blogs published around of the time the 2004 US Presidential election.

11) Airport network [15] $\left(G_{A I R}\right)$ : The Airport network is obtained by analysing routes between all the United States airports in 2010.

The network topologies are shown in Figure 3.

We summarize the properties of the networks used in the experiments in Table I. Given a graph $G$, let $s(G)$ and $d(G)$ be the average shortest path length among all pairs of vertices and the average degree, respectively. Network diameter is the maximum distance between nodes in the network [20]. Network density is a normalized version of the average number of neighbours, which indicates the overall level of interaction between all nodes in a network [21].

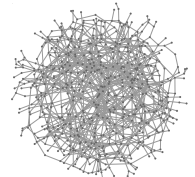

(a) $G_{R}^{0.005}$

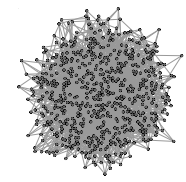

(d) $G_{B A}^{4}$

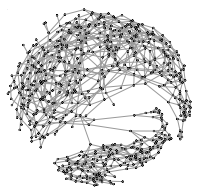

(g) $G_{H}^{4}$

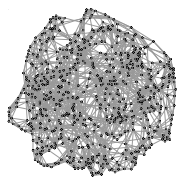

(j) $G_{W S}^{4,0.1}$

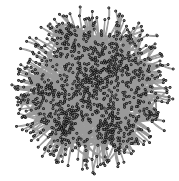

(j) $G_{M A I L}$

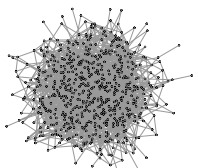

(b) $G_{R}^{0.01}$

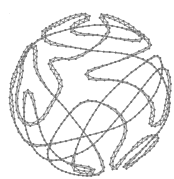

(e) $G_{C}^{2}$

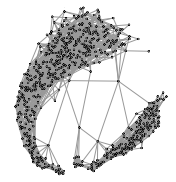

(h) $G_{H}^{8}$

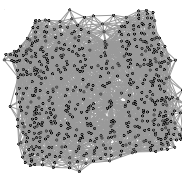

(k) $G_{C A}$

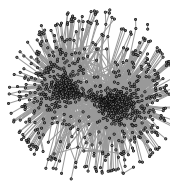

(k) $G_{B L O G}$

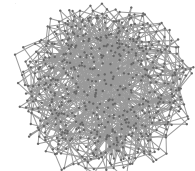

(c) $G_{B A}^{2}$

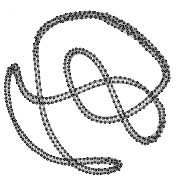

(f) $G_{C}^{4}$

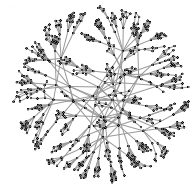

(i) $G_{T S}$

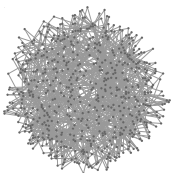

(1) $G_{P R U}^{50,12,2}$

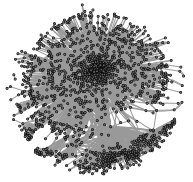

(1) $G_{A I R}$
Fig. 3. The Networks used in the experiments.

For our simulations, starting with an original graph $G$ in Figure 3, at each round (1) we remove $k_{a}$ nodes and their adjacent edges following the attack strategy and then (2) we add $k_{d}$ new nodes such that each of the added nodes is connected with $m$ new edges to $m$ existing nodes. We set $m$ to be the nearest integer rounded from $w \cdot d(G)$ where 


\begin{tabular}{|l|r|r|r|r|r|r|}
\cline { 2 - 7 } \multicolumn{1}{c|}{} & $|V|$ & $|E|$ & Diameter & Density & $d(G)$ & $s(G)$ \\
\hline$G_{R}^{0.005}$ & 584 & 929 & 13 & 0.005 & 3.182 & 5.786 \\
\hline$G_{R}^{0.01}$ & 599 & 1,778 & 7 & 0.010 & 5.937 & 3.796 \\
\hline$G_{B A}^{2}$ & 600 & 1,182 & 7 & 0.007 & 3.940 & 3.986 \\
\hline$G_{B A}^{4}$ & 600 & 2,345 & 5 & 0.013 & 7.817 & 3.033 \\
\hline$G_{C}^{2}$ & 600 & 1,200 & 150 & 0.007 & 4.000 & 75.376 \\
\hline$G_{C}^{4}$ & 600 & 2,400 & 39 & 0.013 & 8.000 & 19.720 \\
\hline$G_{H}^{4}$ & 600 & 1,099 & 11 & 0.006 & 3.663 & 7.318 \\
\hline$G_{H}^{8}$ & 600 & 2,126 & 7 & 0.012 & 7.087 & 4.768 \\
\hline$G_{T S}$ & 600 & 1,228 & 14 & 0.007 & 4.093 & 7.060 \\
\hline$G_{W S}^{4,0.1}$ & 600 & 2,400 & 8 & 0.013 & 8.000 & 4.683 \\
\hline$G_{C A}$ & 600 & 5,401 & 9 & 0.030 & 18.003 & 4.283 \\
\hline$G_{P R U}^{50,12,2}$ & 600 & 1,245 & 7 & 0.007 & 4.143 & 4.075 \\
\hline$G_{M A I L}$ & 1,133 & 10,903 & 8 & 0.009 & 9.622 & 3.606 \\
\hline$G_{B L O G S}$ & 1,224 & 19,025 & - & 0.022 & 27.312 & 2.738 \\
\hline$G_{A I R}$ & 1,574 & 28,236 & - & 0.014 & 21.874 & 3.115 \\
\hline
\end{tabular}

TABLE I

THE PROPERTIES OF THE NETWORKS

$w$ is the edge construction weight. These parameters are summarized in Table II. The aim of the experiments is to evaluate feasibility and usefulness of each strategy and to find the optimal parameter values (e.g. $w$ ) of each strategy at the same time.

\begin{tabular}{||c|l||}
\hline \hline Parameters & Description \\
\hline$k_{a}$ & The number of the removed nodes in an attack phase \\
\hline$k_{d}$ & The number of the added nodes in a defense phase \\
\hline$w$ & The edge construction weight \\
\hline$d(G)$ & The average degree in a graph $G$ \\
\hline$m$ & $\begin{array}{l}\text { The number of the added edges per node in a defense } \\
\text { phase: } \operatorname{Round}(w \cdot d(G))\end{array}$ \\
\hline
\end{tabular}

TABLE II

SUMMARY OF PARAMETERS IN SIMULATION. WHEN $k_{a}=k_{d}$ AND $w=1.0$, AN ATTACKER'S DAMAGE ABILITY IS APPROXIMATELY EQUAL TO A DEFENDER'S REPAIR ABILITY IN TERMS OF THE NUMBER OF CONNECTIONS

With fixed $k_{a}, k_{d}$, and $m$, we can observe how the size of the largest connected component and the average degree in a graph evolve. For example, with $k_{a}=k_{d}=10$ and $w=1.0$, Figure 4 shows how these values in $G_{R}^{0.005}$ are changed under iterated attack and defense operations. The size of the largest connected component in each round is normalized by dividing by the size of the largest connected component in the original graph. From this figure, we can see that $\mathbf{D}^{\text {balance }}$ only performed well against $\mathbf{A}^{\text {degree }}$ or $\mathbf{A}^{\text {central}}$ : the size of the largest connected component in the graph remained unchanged during the 100 rounds against these attacks while $\mathbf{D}^{\text {random }}$ and $\mathbf{D}^{\text {prefer }}$ are not effective - within 40 rounds the size of the largest connected component has fallen by a half. Interestingly, there exists a relationship between the size of the largest connected component and the average degree as we discussed in Section IV: The size of the largest connected component in $G_{R}^{0.005}$ started to drop dramatically when the average degree of the network falls below 2 .

In this paper, our research interest is finding the best attack and defense strategies with varying $k_{a}, k_{d}$, and $w$ rather than observing how network connectivity evolves in a particular network with fixed $k_{a}, k_{d}$, and $w$. Even if an attacker finds
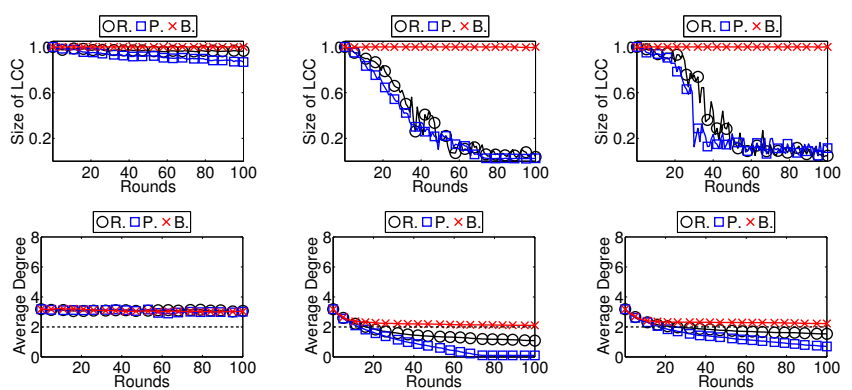

(a) $\mathbf{A}^{\text {random }}$

(b) $\mathbf{A}^{\text {degree }}$

(c) $\mathbf{A}^{\text {central }}$

Fig. 4. Changes in the size of the largest connected component and the average degree in $G_{R}^{0.005}$ over rounds. The first row graphs show the changes in the size of the largest connected component and the second row graphs show the changes in the average degree.

the ideal attack strategy for a given budget $k_{a}$, a defender may block this attack even with a naive defense strategy if she can increase $k_{d}$ and/or $w$ without limit. In this context, an attacker's goal should be interpreted to find an attack strategy maximizing the defense costs $\left(k_{d}\right.$ and/or $\left.w\right)$ while the defender wishes to find a defense strategy minimizing them.

With the edge construction weight $w$ ranging from 0.5 to 1.5 , we first discuss the effects of $w$ for each of the fifteen graphs in Figure 3. To demonstrate this we fix $k_{a}=k_{d}=10$ and analyse the size of the largest connected component and the average degree in each graph after the 100th round. The experimental results for each network are shown, respectively, in Figure 5, 6, 7, 8, 9, 10, 11, 12, 13, 14, 15, 16, 17, 18, and 19. The results for best attack and defense strategies with $w$ are summarized in Table III.

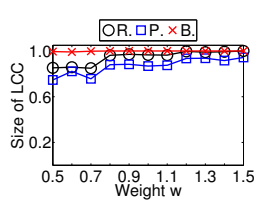

OR. IP. $\times$ B.

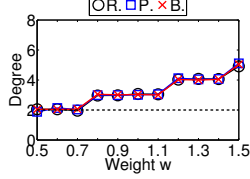

(a) $\mathbf{A}^{\text {random }}$

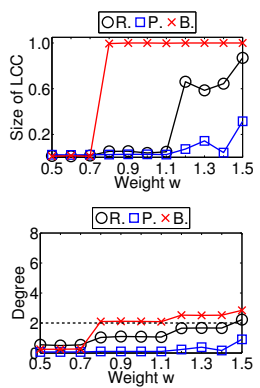

(b) $\mathbf{A}^{\text {degree }}$

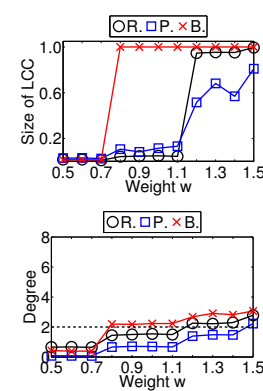

(c) $\mathbf{A}^{\text {central }}$
Fig. 5. Random graph 1 with w: changes in the size of the largest connected component in $G_{R}^{0.005}$ with $w$. In this figure, and in figures 6 through 16 , the first row graphs show the changes in the size of the largest connected component and the second row graphs shows the changes in the average degree.

From these figures, we can see $\mathbf{D}^{\text {balance }}$ performed well except for the case against $\mathbf{A}^{\text {central }}$ in $G_{T S}$ (see Figure 13(c)). When a defender uses $\mathbf{D}^{\text {balance }}$ even with a small $w \leq 1.0$, most nodes in all networks except for $G_{T S}$ and $G_{P R U}^{50,12,2}$ remain connected to each other. However, $\mathbf{D}^{\text {random }}$ and $\mathbf{D}^{\text {prefer }}$ are not sufficiently effective against $\mathbf{A}^{\text {degree }}$ or $\mathbf{A}^{\text {central }}$ in many network topologies. For example, when $w=1.0$, the graph $G_{R}^{0.005}$ is totally disconnected if $\mathbf{D}^{\text {random }}$ or $\mathbf{D}^{\text {prefer }}$ is used against either $\mathbf{A}^{\text {degree }}$ or $\mathbf{A}^{\text {central }}$ (see Figure 5(b) and (c)). In fact, even when $w=1.5$, there are not enough edges to defend against $\mathbf{A}^{\text {degree }}$ (see Figure 5(b)). In particular, $\mathbf{D}^{\text {prefer }}$ 


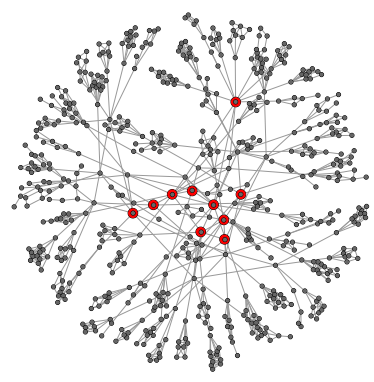

(a)

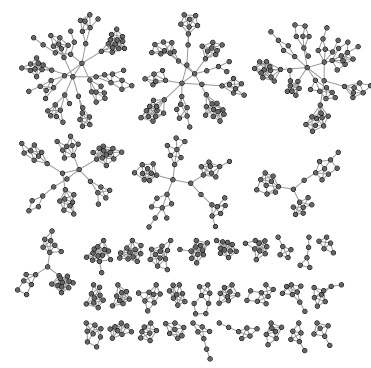

(b)

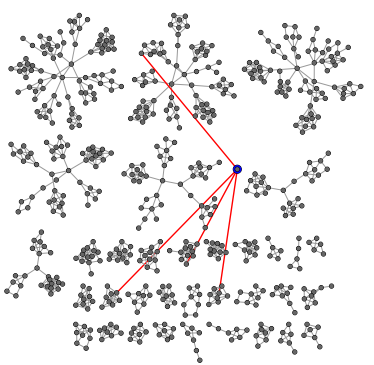

(c)

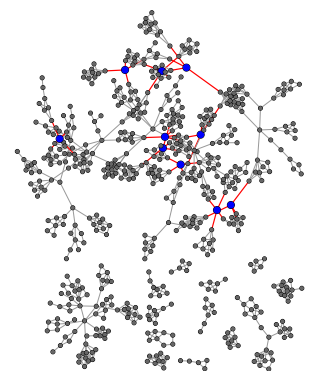

(d)

Fig. 20. Performing $\mathbf{A}^{\text {central }}$ and $\mathbf{D}^{\text {balance }}$ on $G_{T S}$ in the first round in the transit-stub graph. (a) The ten nodes (large circle) are selected to be removed for $\mathbf{A}^{\text {central }}$. (b) The graph is totally disconnected after the first attack. (c) A new node and its connections are added by $\mathbf{D}^{\text {balance }}$. This node plays a role as the node connecting different clusters. (d) The ten nodes and their connections are newly created after the first defense. However, many nodes are still disconnected from the largest connected component (the above subnetwork) in the graph.

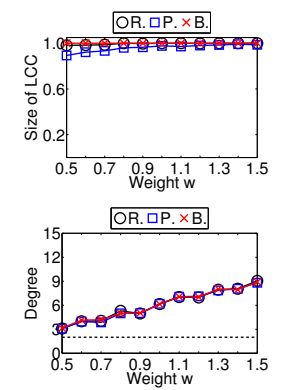

(a) $\mathbf{A}^{\text {random }}$
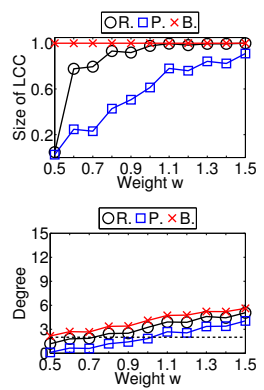

(b) $\mathbf{A}^{\text {degree }}$
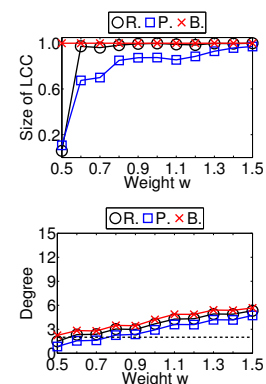

(c) $\mathbf{A}^{\text {central }}$

Fig. 6. Random graph 2 with w: changes in the size of the largest connected component in $G_{R}^{0.01}$ with $w$.

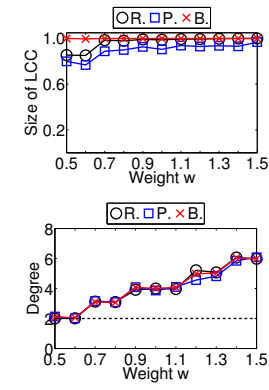

(a) $\mathbf{A}^{\text {random }}$
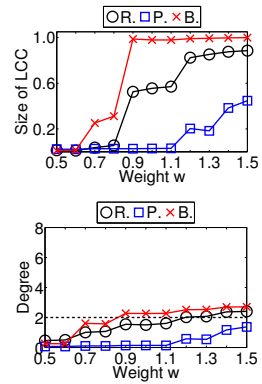

(b) $\mathbf{A}^{\text {degree }}$
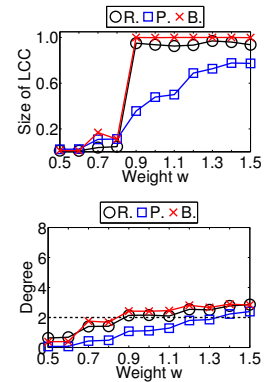

(c) $\mathbf{A}^{\text {central }}$

Fig. 7. Scale-free graph 1 with w: changes in the size of the largest connected component in $G_{B A}^{2}$ with $w$.

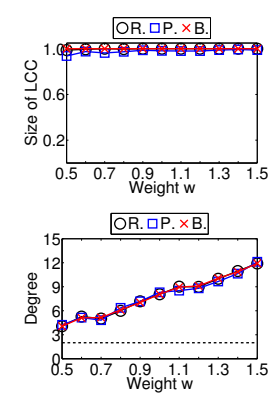

(a) $\mathbf{A}^{\text {random }}$
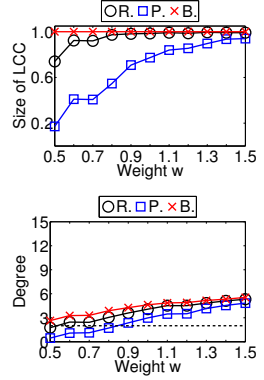

(b) $\mathbf{A}^{\text {degree }}$
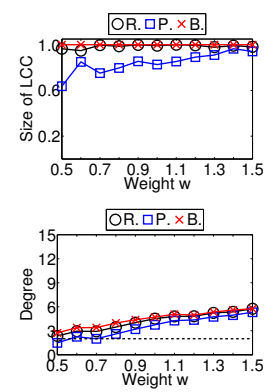

(c) $\mathbf{A}^{\text {central }}$

Fig. 8. Scale free graph 2 with w: changes in the size of the largest connected component in $G_{B A}^{4}$ with $w$.
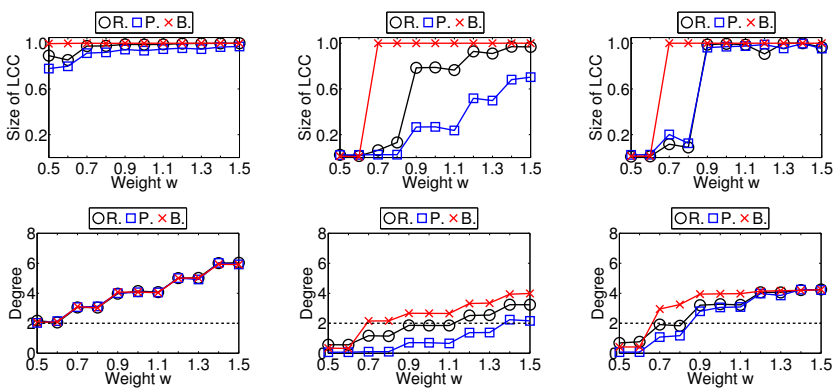

(a) $\mathbf{A}^{\text {random }}$

(b) $\mathbf{A}^{\text {degree }}$

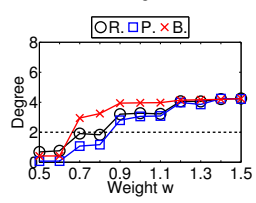

(c) $\mathbf{A}^{\text {central }}$

Fig. 9. Chord graph 1 with w: changes in the size of the largest connected component in $G_{C}^{2}$ with $w$.

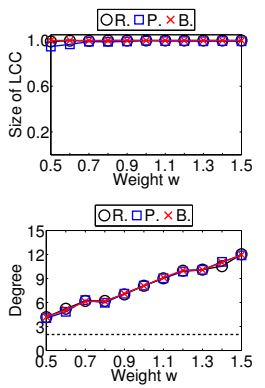

(a) $\mathbf{A}^{\text {random }}$
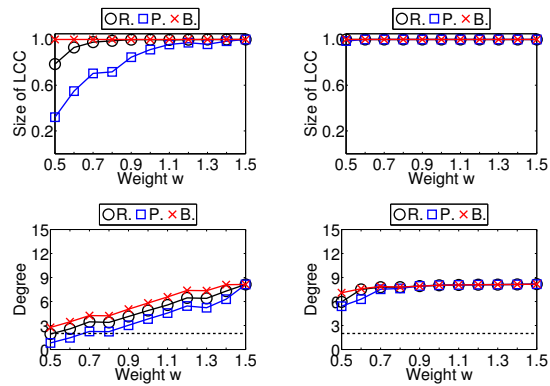

(b) $\mathbf{A}^{\text {degree }}$

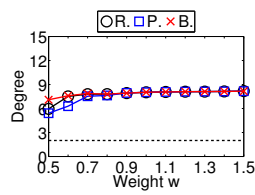

(c) $\mathbf{A}^{\text {central }}$

Fig. 10. Chord graph 2 with w: changes in the size of the largest connected component in $G_{C}^{4}$ with $w$.

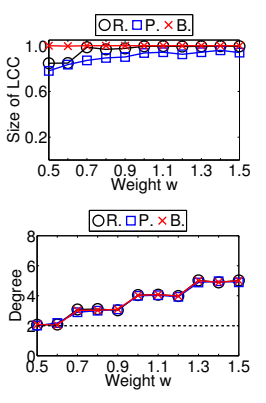

(a) $\mathbf{A}^{\text {random }}$
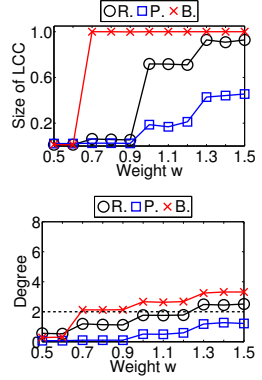

(b) $\mathbf{A}^{\text {degree }}$

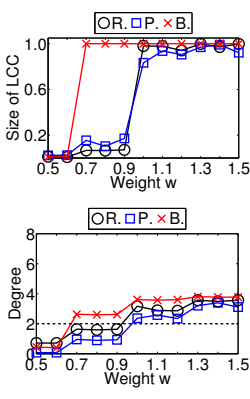

(c) $\mathbf{A}^{\text {central }}$

Fig. 11. Hypergraph 1 with w: changes in the size of the largest connected component in $G_{H}^{4}$ with $w$. 


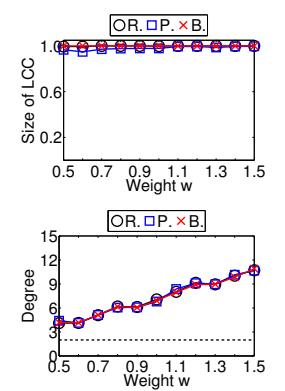

(a) $\mathbf{A}^{\text {random }}$
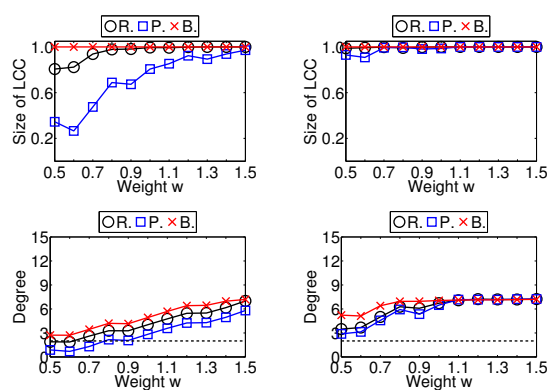

(b) $\mathbf{A}^{\text {degree }}$

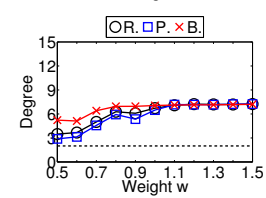

(c) $\mathbf{A}^{\text {central }}$

Fig. 12. Hypergraph 2 with w: changes in the size of the largest connected component in $G_{H}^{8}$ with $w$.
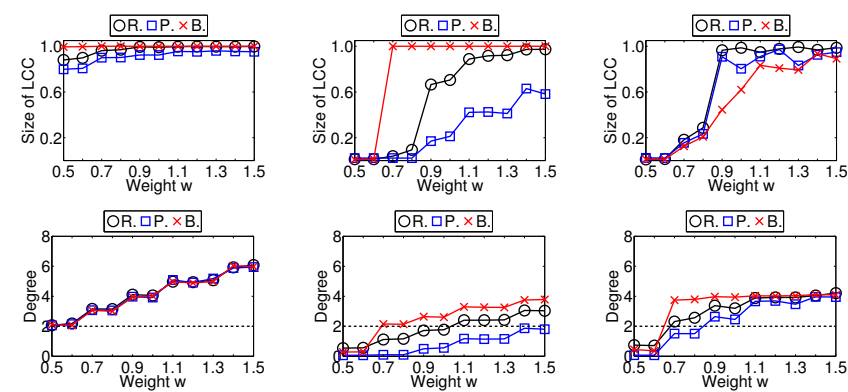

(a) $\mathbf{A}^{\text {random }}$
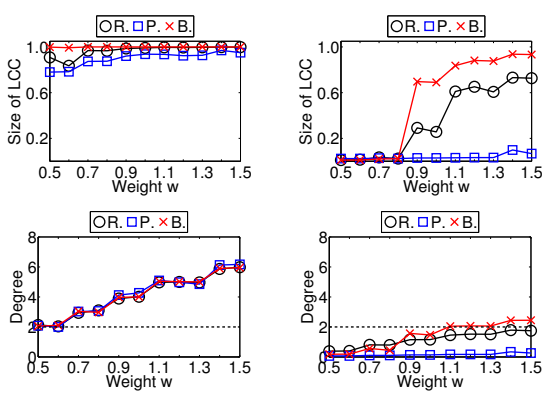

(a) $\mathbf{A}^{\text {random }}$
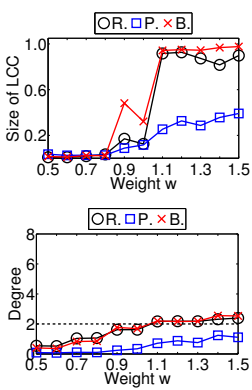

(b) $\mathbf{A}^{\text {degree }}$

(c) $\mathbf{A}^{\text {central }}$

Fig. 16. PRU graph with $\mathrm{w}$ : changes in the size of the largest connected component in $G_{P R U}^{50,12,2}$ with $w$.
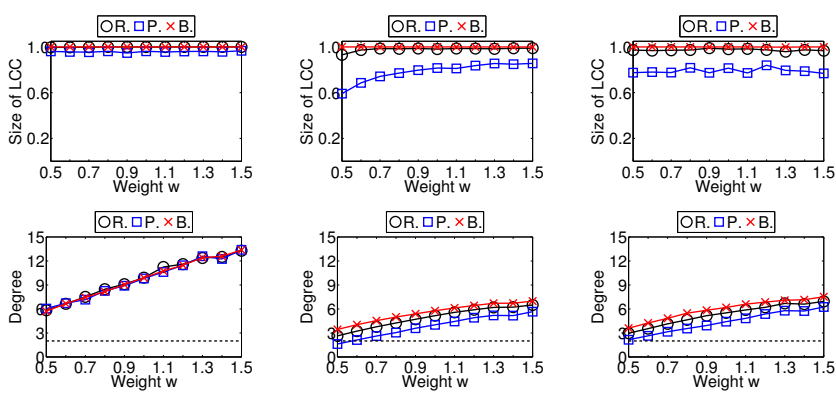

(a) $\mathbf{A}^{\text {random }}$

(b) $\mathbf{A}^{\text {degree }}$

(c) $\mathbf{A}^{\text {central }}$

Fig. 17. Email network with w: changes in the size of the largest connected component in $G_{M A I L}$ with $w$.

connected component in $G_{T S}$ with $w$.
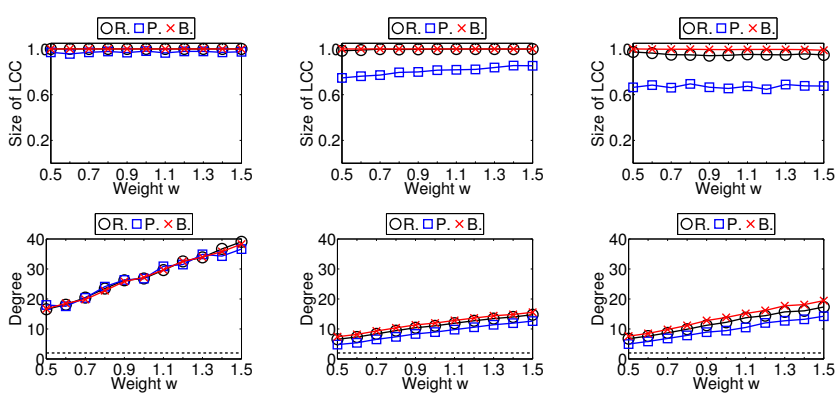

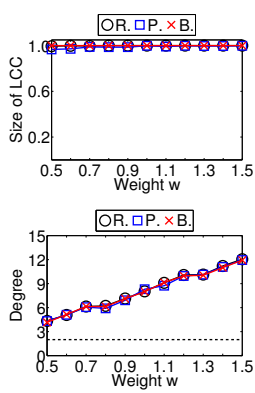

(a) $\mathbf{A}^{\text {random }}$
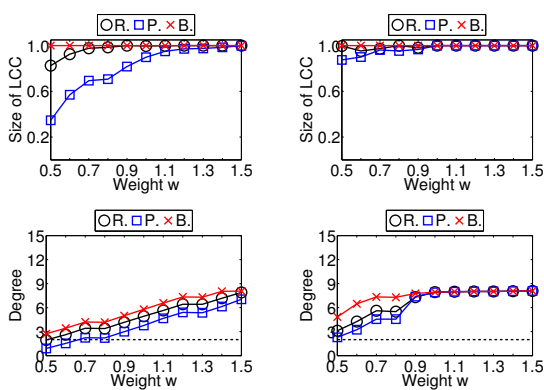

(b) $\mathbf{A}^{\text {degree }}$

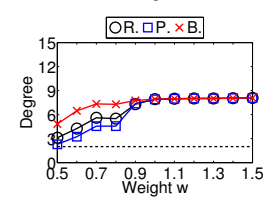

(c) $\mathbf{A}^{\text {central }}$

Fig. 14. Small-world graph with w: changes in the size of the largest connected component in $G_{W S}^{4,0.1}$ with $w$.
(a) $\mathbf{A}^{\text {random }}$
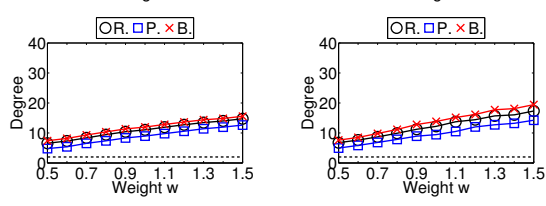

(b) $\mathbf{A}^{\text {degree }}$

(c) $\mathbf{A}^{\text {central }}$

Fig. 18. Blog network with w: changes in the size of the largest connected component in $G_{B L O G}$ with $w$.

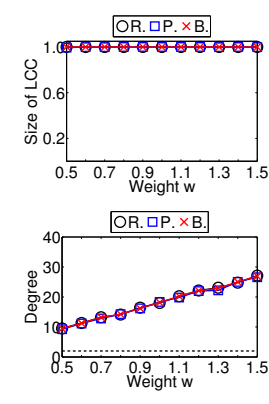

(a) $\mathbf{A}^{\text {random }}$
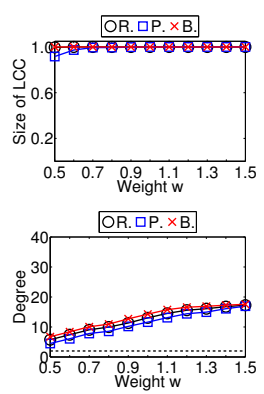

(b) $\mathbf{A}^{\text {degree }}$
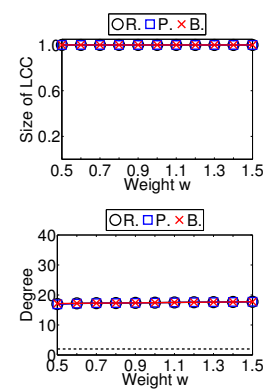

(c) $\mathbf{A}^{\text {central }}$

Fig. 15. CAN with w: changes in the size of the largest connected component in $G_{C A}$ with $w$.

performed badly against $\mathbf{A}^{\text {degree }}$. Since most real networks exhibit preferential connectivity [6], a real-world network may be very vulnerable to high-degree node attacks even if the
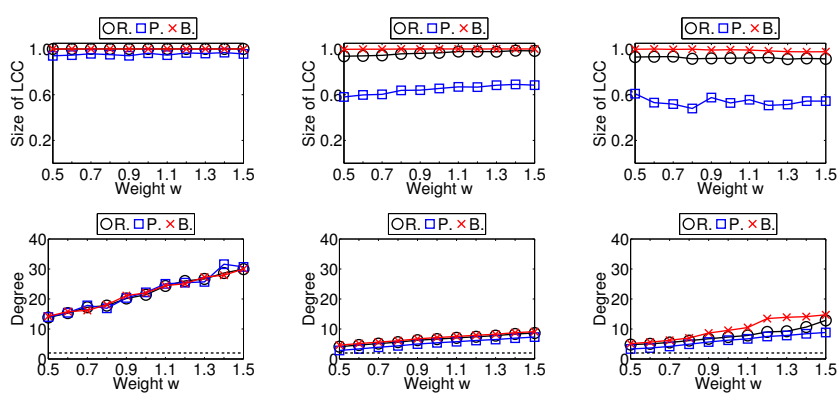

(a) $\mathbf{A}^{\text {random }}$

(b) $\mathbf{A}^{\text {degree }}$

(c) $\mathbf{A}^{\text {central }}$

Fig. 19. Airport network with w: changes in the size of the largest connected component in $G_{A I R}$ with $w$.

network has grown continuously up with new nodes over time. Our results on real networks supported this conjecture (see Figure 17(b), 18(b), 19(b)). 


\begin{tabular}{|l|c|c|c|c|}
\cline { 2 - 5 } \multicolumn{1}{c|}{} & Best Attack & Best Defense & $w$ & Damage \\
\hline$G_{R}^{0.005}$ & $\mathbf{A}^{\text {degree }}, \mathbf{A}^{\text {central }}$ & $\mathbf{D}^{\text {balance }}$ & 0.8 & None \\
\hline$G_{R}^{0.01}$ & - & $\mathbf{D}^{\text {balance }}$ & 0.5 & None \\
\hline$G_{B A}^{2}$ & $\mathbf{A}^{\text {degree }}, \mathbf{A}^{\text {central }}$ & $\mathbf{D}^{\text {balance }}$ & 0.9 & None \\
\hline$G_{B A}^{4}$ & - & $\mathbf{D}^{\text {balance }}$ & 0.5 & None \\
\hline$G_{C}^{2}$ & $\mathbf{A}^{\text {degree }}, \mathbf{A}^{\text {central }}$ & $\mathbf{D}^{\text {balance }}$ & 0.7 & None \\
\hline$G_{C}^{4}$ & - & $\mathbf{D}^{\text {balance }}$ & 0.5 & None \\
\hline$G_{H}^{4}$ & $\mathbf{A}^{\text {degree }}, \mathbf{A}^{\text {central }}$ & $\mathbf{D}^{\text {balance }}$ & 0.7 & None \\
\hline$G_{H}^{8}$ & - & $\mathbf{D}^{\text {balance }}$ & 0.5 & None \\
\hline$G_{T S}$ & $\mathbf{A}^{\text {central }}$ & $\mathbf{D}^{\text {random }}$ & 0.9 & Small \\
\hline$G_{W S}^{4,0.1}$ & - & $\mathbf{D}^{\text {balance }}$ & 0.5 & None \\
\hline$G_{C A}$ & - & $\mathbf{D}^{\text {random }}, \mathbf{D}^{\text {balance }}$ & 0.5 & None \\
\hline$G_{P R U}^{50,12,2}$ & $\mathbf{A}^{\text {degree }}$ & $\mathbf{D}^{\text {balance }}$ & 1.5 & Small \\
\hline$G_{M A I L}$ & - & $\mathbf{D}^{\text {balance }}$ & 0.5 & None \\
\hline$G_{B L O G}$ & - & $\mathbf{D}^{\text {balance }}$ & 0.5 & None \\
\hline$G_{A I R}$ & - & $\mathbf{D}^{\text {balance }}$ & 0.5 & None \\
\hline
\end{tabular}

TABLE III

THE BEST ATTACK AND DEFENSE STRATEGIES FOR NETWORKS

On the other hand, $\mathbf{A}^{\text {random }}$ is not effective from the attacker's point of view; the size of the largest connected component remained unchanged and the average degree is still greater than 2 after 100 rounds if any defense strategy is used with $w=1.0$. This is natural enough; the damage done by random failures is not essentially greater than the level of repair by random replenishment. So network connectivity will be maintained well on most popular network topologies under random node failure or removal if the same number of new nodes can be continuously recruited.

To maintain network connectivity, a possible approach is to increase network density. In our experiments, the half of network topologies with a high network density $\geq 0.009\left(G_{R}^{0.01}\right.$, $G_{B A}^{4}, G_{C}^{4}, G_{H}^{8}, G_{W S}^{4,0.1}, G_{C A}, G_{M A I L}, G_{B L O G}$, and $G_{A I R}$ — see Table I) is resilient against any attack strategies when $\mathbf{D}^{\text {balance }}$ is used with $w=0.5$ only (see Figure $6,8,10,12,14$ and 15). In fact, $\mathbf{D}^{\text {random }}$ is also adequate in these networks except $G_{B L O G}$ and $G_{A I R}$ if a defender can increase $w$ to 1.0. For the two real networks, $G_{B L O G}$ and $G_{A I R}, \mathbf{D}^{\text {random }}$ is not perfect against $\mathbf{A}^{\text {central }}$ even with a large $w=1.5$.

Interestingly, $\mathbf{D}^{\text {balance }}$ is worse than the other defense strategies against $\mathbf{A}^{\text {central }}$ in $G_{T S}$ (see Figure 13(c)). This is because a small number of nodes connect different clusters in $G_{T S}$. Since these nodes have high betweenness centrality, transit-stub graphs are inherently very vulnerable to $\mathbf{A}^{\text {central }}$. Unfortunately, $\mathbf{D}^{\text {balance }}$ cannot change this weakness of transit-stub graphs since nodes newly recruited in the defense phase generally play a role as new gateway nodes by connecting separated clusters. This trend can be observed in Figure 20.

Another interesting observation is the relationship between the size of the largest connected component and the average degree in a graph. As we discussed in Section IV, in all experiments, we can see that the size of the largest connected component is not maintained well when the average degree of the network falls below 2 regardless of defense strategy, but the opposite is not true (see the counter example in Figure 13(c)).

Finally, we discuss how the performance of attack and defense strategies may change when $k_{a} \neq k_{d}$. As $k_{d}$ increases, network connectivity between nodes will increase over rounds. Figures 21 (against high-degree node attacks) and 22 (against betweenness centrality attacks) show the effects of varying $k_{d}$ from 7 to 13 with $k_{a}=10$ and $w=1.0$. To demonstrate this we plot the size of the largest connected component in a graph at the 50th round.

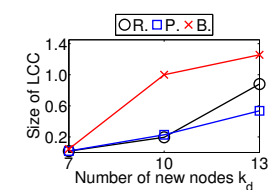

(a) $G_{R}^{0.005}$

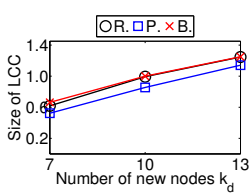

(d) $G_{B A}^{4}$

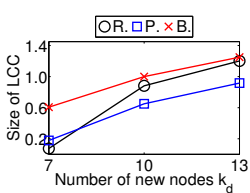

(g) $G_{H}^{4}$

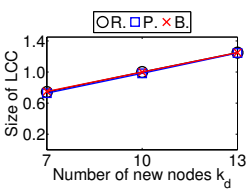

(j) $G_{W S}^{4,0.1}$

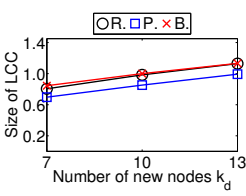

(m) $G_{M A I L}$

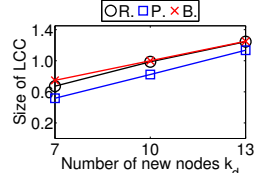

(b) $G_{R}^{0.01}$

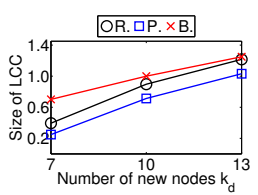

(e) $G_{C}^{2}$

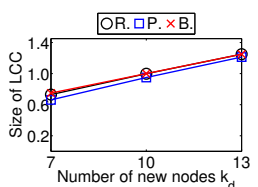

(h) $G_{H}^{8}$

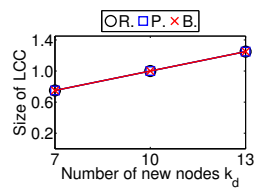

(k) $G_{C A}$

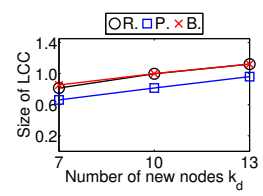

(n) $G_{B L O G}$

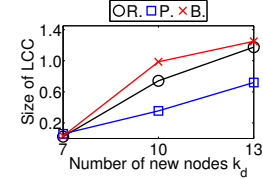

(c) $G_{B A}^{2}$

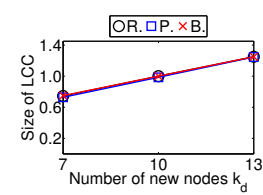

(f) $G_{C}^{4}$

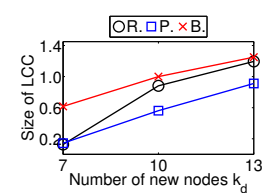

(i) $G_{T S}$
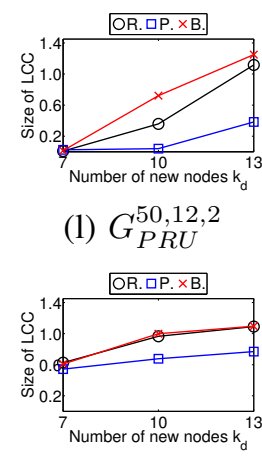

(o) $G_{A I R}$
(1) $G_{P R U}^{50,12,2}$

Fig. 21. The size of the largest connected component with $k_{d}$ against highdegree node attacks.

As $k_{d}$ increases, so does the size of the largest connected component. This is natural enough, and is particularly clear in low density networks $\left(G_{R}^{0.005}, G_{B A}^{2}, G_{C}^{2}, G_{H}^{4}, G_{T S}\right.$ and $\left.G_{P R U}^{50,12,2}\right)$.

The performance of $\mathbf{D}^{\text {balance }}$ is still better than those of the other two defense strategies and is highly scalable in terms of $k_{d}$ : the gap between them is clearly shown in low density networks when $k_{d}>k_{a}$ and in some networks $\left(G_{R}^{0.01}, G_{B A}^{4}\right.$, $G_{C}^{2}, G_{H}^{4}$ and $\left.G_{T S}\right)$ when $k_{d}<k_{a}$. Interestingly, $\mathbf{D}^{\text {balance }}$ produced the best result in $G_{T S}$ against $\mathbf{A}^{\text {central }}$ when $k_{d}>$ $k_{a}$; it is different from the case when $k_{d}=k_{a}$.

\section{Conclusions}

Barabási, Albert and Jeong showed that while small-world networks were resilient against random node failure, they were very vulnerable to targeted attacks. Nagaraja and Anderson extended this single-shot analysis to the dynamic case, so that 


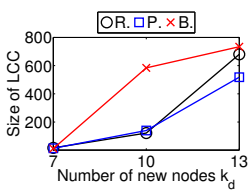

(a) $G_{R}^{0.005}$

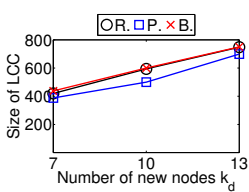

(d) $G_{B A}^{4}$

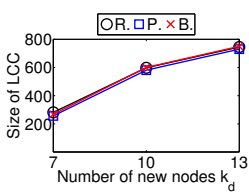

(g) $G_{H}^{4}$

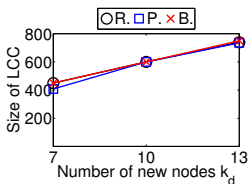

(j) $G_{W S}^{4,0 .}$

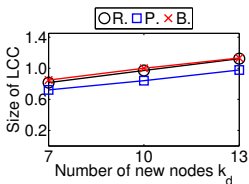

(m) $G_{M A I L}$

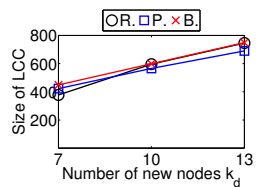

(b) $G_{R}^{0.01}$

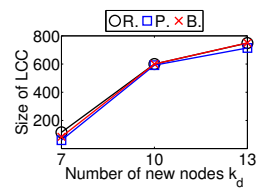

(e) $G_{C}^{2}$

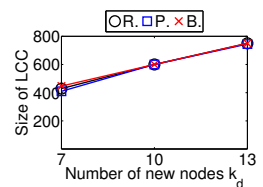

(h) $G_{H}^{8}$

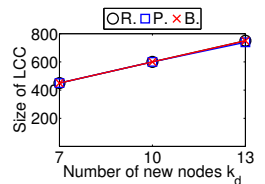

(k) $G_{C A}$

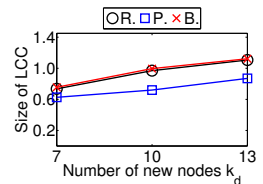

(n) $G_{B L O G}$

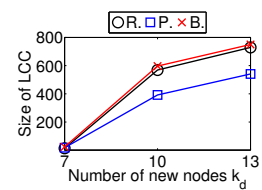

(c) $G_{B A}^{2}$

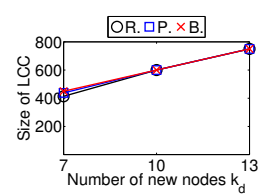

(f) $G_{C}^{4}$

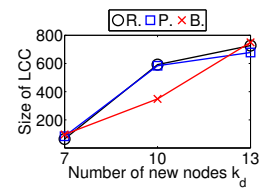

(i) $G_{T S}$

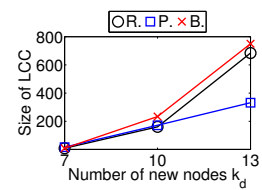

(1) $G_{P R U}^{50,12,2}$

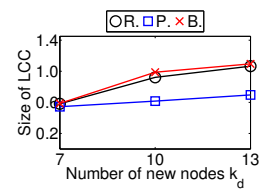

(o) $G_{A I R}$
Fig. 22. The size of the largest connected component with $k_{d}$ against betweenness centrality attacks.

attack and defense strategies could be measured against each other. We have extended their work to a wide range of network topologies including some real-world networks, and to account for the costs of replacing edges as well as nodes. In summary,

- the best defense strategy in general is balanced replenishment, $\mathbf{D}^{\text {balance }}$. For high density networks with network density $\geq 0.009$, it is enough to set $w=0.5$.

- the best attack strategy in general targets vertex order or betweenness centrality, that is $\mathbf{A}^{\text {degree }}$ or $\mathbf{A}^{\text {central }}$, in the sense that it maximizes the cost of defense. However, when the network has a hierarchical tree-like structure, it will often be better to use $\mathbf{A}^{\text {central }}$.

- it is necessary but not sufficient for the defender to maintain the average node degree $\geq 2$ to maintain connectivity.

- a real-world network may be very vulnerable to $\mathbf{A}^{\text {degree }}$ or $\mathbf{A}^{\text {central }}$ even if the network has grown continuously up with new nodes and connections over time.

In future work, we plan to develop better models of the adversary. We may consider not only an adversary with global knowledge of network topology but also a weaker adversary with limited information (e.g. a local police force). For example, we expect that $\mathbf{D}^{\text {random }}$ is secure against any adversary with no knowledge of the network topology at all; what strategies suffice against an attacker whose knowledge is is local? Also, while adding more edges to a network may be a viable strategy for a disease pathogen, it may not help an insurgent group as a better-connected network may be more vulnerable to insider threats.

As an extension to this work, we plan to consider a theoretical study to formally generalize and verify our results. We will also employ more advanced centrality metrics such as bridging centrality [22] to improve the performance of strategies.

\section{ACKNOWLEDGMENT}

The first author's research is funded by Northrop Grumman Systems Corporation. The contents of this article do not necessarily express the views of Northrop Grumman Systems Corporation. Authors also acknowledge support from FKPPL Internal Associated Laboratory for grid computing resources.

\section{REFERENCES}

[1] S. Boccaletti, V. Latora, Y. Moreno, M. Chavez, and D. Hwang, "Complex networks: Structure and dynamics," Physics Reports, vol. 424, no. 4-5, pp. 175-308, February 2006.

[2] M. E. J. Newman, A. L. Barabási, and D. J. Watts, Eds., The Structure and Dynamics of Networks. Princeton University Press, 2010.

[3] R. Albert, H. Jeong, and A.-L. Barabási, "Error and attack tolerance of complex networks," Nature, vol. 406, no. 6794, pp. 378-382, July 2000.

[4] S. Nagaraja and R. Anderson, "The topology of covert conflict," in Proceedings of the 5th Workshop on The Economics of Information Security (WEIS 2006), 2006.

[5] R. Axelrod and W. D. Hamilton, "The evolution of cooperation," Science, vol. 211, pp. 1390-1396, 1981.

[6] A.-L. Barabási and R. Albert, "Emergence of Scaling in Random Networks," Science, vol. 286, no. 5439, pp. 509-512, 1999.

[7] I. Stoica, R. Morris, D. Liben-Nowell, D. R. Karger, M. F. Kaashoek, F. Dabek, and H. Balakrishnan, "Chord: a scalable peer-to-peer lookup protocol for internet applications," IEEE/ACM Transactions on Networking, vol. 11, no. 1, pp. 17-32, 2003.

[8] F. Saffre and R. Ghanea-Hercock, "Beyond anarchy: self-organized topology for peer to peer networks," Complexity, vol. 9, no. 2, pp. 49-53, 2003.

[9] K. Calvert, M. Doar, and E. Zegura, "Modeling Internet Topology," Communications Magazine, IEEE, vol. 35, no. 6, pp. 160-163, Jun 1997.

[10] D. J. Watts, Small Worlds: The Dynamics of Networks between Order and Randomness (Princeton Studies in Complexity), illustrated edition ed. Princeton University Press, November 2003.

[11] S. Ratnasamy, P. Francis, M. Handley, R. Karp, and S. Schenker, "A Scalable Content-Addressable Network," in SIGCOMM '01: Proceedings of the 2001 conference on Applications, technologies, architectures, and protocols for computer communications. New York, NY, USA: ACM, 2001, pp. 161-172.

[12] G. Pandurangan, P. Raghavan, and E. Upfal, "Building low-diameter peer-to-peer networks," Selected Areas in Communications, IEEE Journal on, vol. 21, no. 6, pp. 995 - 1002, aug. 2003.

[13] R. Guimerà, L. Danon, D. A. Guilera, F. Giralt, and A. Arenas, "Self-similar community structure in a network of human interactions," Physical Review E, vol. 68, no. 6, Dec 2003.

[14] L. A. Adamic and N. Glance, "The political blogosphere and the 2004 u.s. election: divided they blog," in Proceedings of the 3rd international workshop on Link discovery, ser. LinkKDD '05. New York, NY, USA: ACM, 2005, pp. 36-43.

[15] T. Opsahl, "Why Anchorage is not (that) important: Binary ties and Sample selection," 2011. [Online]. Available: http://toreopsahl.com/2011/08/12/why-anchorage-is-not-thatimportant-binary-ties-and-sample-selection/

[16] P. Holme, B. J. Kim, C. N. Yoon, and S. K. Han, "Attack vulnerability of complex networks," Physical Review E, vol. 65, no. 5, p. 056109, May 2002.

[17] L. Zhao, K. Park, and Y.-C. Lai, "Attack vulnerability of scale-free networks due to cascading breakdown," Physical Review E, vol. 70, no. 3, p. 035101, Sep 2004. 
[18] J. Domingo-Ferrer and Úrsula González-Nicolás, "Decapitation of networks with and without weights and direction: The economics of iterated attack and defense," Computer Networks, vol. 55, no. 1, pp. 119-130, 2011.

[19] U. Brandes, "A faster algorithm for betweenness centrality," Journal of Mathematical Sociology, vol. 25, pp. 163-177, 2001.

[20] H. Per and H. Frank, "Eccentricity and Centrality in Networks," Social Networks, vol. 17, no. 1, pp. 57-63, 1995.

[21] J. Dong and S. Horvath, "Understanding Network Concepts in Modules," BMC Systems Biology, vol. 1, no. 1, 2007.

[22] W. Hwang, T. Kim, M. Ramanathan, and A. Zhang, "Bridging centrality: graph mining from element level to group level," in Proceedings of the 14th ACM SIGKDD international conference on Knowledge discovery and data mining, ser. KDD '08. New York, NY, USA: ACM, 2008, pp. 336-344. 